\title{
Space Charge Behaviour in LDPE after AC Electrical Ageing
}

\author{
M. Fu ${ }^{1 *}$, G. Chen ${ }^{2}$ and X. Liu $^{3}$ \\ ${ }^{1}$ Department of Engineering, University of Leicester, Leicester, LE2 7RH, UK \\ ${ }^{2}$ School of Electronics and Computer Science, University of Southampton, Southampton, SO17 1BJ, UK \\ ${ }^{3}$ State Key Laboratory of Electrical Insulation, Xian Jiaotong University, Xian, China \\ *E-mail:mf75@le.ac.uk
}

\begin{abstract}
Space charge accumulation after ac ageing and its effect on dc poling has been studied in lowdensity polyethylene (LDPE). LDPE film samples with thickness of around $180 \mu \mathrm{m}$ were aged at an electric stress of $50 \mathrm{kV} / \mathrm{mm}$ under $50 \mathrm{~Hz}$ ac voltage for various times at ambient temperature. Space charge dynamics in the samples after ageing were monitored using the pulsed electroacoustic (PEA) technique. Except the presence of considerable amount of space charge accumulation in the aged sample, the ageing has also brought a significant influence into the space dynamic under dc poling voltage. The ageing extent was previously investigated by chemical analysis (i.e. Fourier Transform Infrared FTIR and Raman spectrum) revealed little change taken place in the bulk of the material after ac ageing in the time frame studied. This indicates that the space charge measurement may be an effective method for diagnosing the early stage of material deterioration. It also provides additional information about charge mobility and trap depth that can be extracted from space charge profile.
\end{abstract}

\section{INTRODUCTION}

Over last two decades, numerous researches have been carried out to develop further a fundamental understanding of the build up of trapped space charge within solid dielectric materials [1-9] which has resulted in a better understanding of charge dynamics and their effect on material selection and processing. However, majority of the work was focused on the issue of the application of polymeric material under dc conditions; little attention has been given to the dynamics of space charge under ac voltage and the effect of ac ageing process on space charge formation in the bulk insulation [6-12]. It is believed that under ac conditions the changes in polarity every half-cycle limits the time of charge trapping and accumulating when ac frequency is greater than $0.01 \mathrm{~Hz}$ [10]. Recent work using other techniques $[6-8,11,12]$ demonstrated that the presence of space charge in polymeric cable insulation materials under ac conditions. However, no quantitative charge distributions were presented. Additionally, it is believed that space charge build up can, under certain conditions, be a contributing factor to electrical tree initiation of ac high voltage insulation[13]. As the majority of XLPE $\mathrm{HV}$ cables will be operating under ac conditions in the immediate future, it is appropriate to investigate charge trapping and mobility under such conditions.
Moreover, a large amount of polymer insulated underground power cables are approaching to the end of their design life after nearly 20 years operation. Because replacement costs are high it is important to be able to evaluate the integrity of the insulation (its reliability) and predict the remnant of life of this valuable asset. This requires both diagnostic techniques and life expressions, both of which require a physical understanding of the mechanism of thermo-electric ageing for their validation.

This paper reports on some primarily results from the attempt to use space charge measurement to assess ageing extent of LDPE. Samples were subjected to 50 $\mathrm{Hz}$ ac electric stresses in the range of $50 \mathrm{kV} / \mathrm{mm}$ at room temperature for a period of time. Space charge accumulation at different ageing times was monitored using the pulsed electro-acoustic (PEA) technique. The influence of electrical ageing on space charge behaviour under dc poling voltage has also been investigated; the results suggested that space charge measurement is able to provide valuable information for polymer insulation degradation assessment.

\section{AC ELECTRICAL AGEING}

Space charge formation in a material is greatly affected by the presence of impurities and additives as they can act either as ionisable centre under a high electric stress or as trapping sites. In order to reduce the influence of the impurities, additive-free low-density polyethylene (LDPE) was selected for the present study because of its relatively simple chemical structure and its wide applications in cable insulation. The LDPE film of the sample was about $180 \mu \mathrm{m}$ thick.

$\mathrm{AC}$ ageing was carried out at ambient temperature for various periods of time at electric stress of $50 \mathrm{kV} / \mathrm{mm}$. In order to prevent from flashover the top brass electrode was cast in epoxy resin and the bottom electrode was semicon.

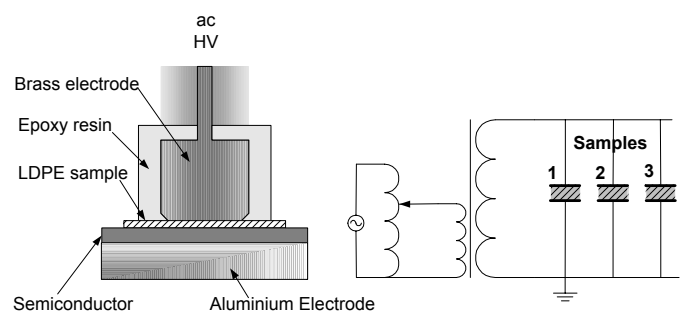

Fig. 1. Schematic diagram of sample and testing circuit. 


\section{EXPERIMENTAL RESULTS}

\section{Space Charge Accumulation over AC Ageing}

Once the samples were removed from the ageing rig after a variety of ageing period of time, space charge accumulation was examined using the PEA method. The typical charge distributions for different periods of ageing time are presented in Fig. 2.

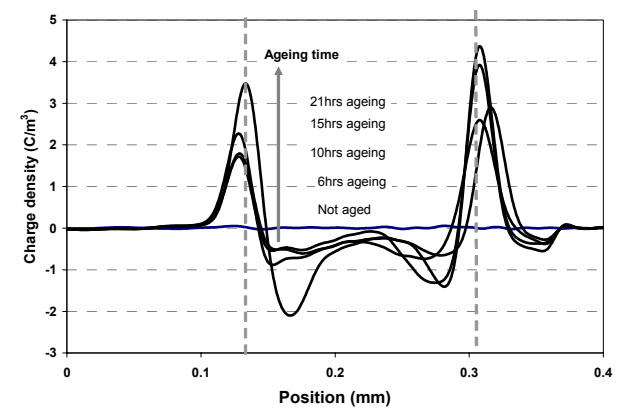

Fig. 2. Charge distribution in LDPE aged for different period of time at $50 \mathrm{kV} / \mathrm{mm}$.

It is noticed that negative charge was formed in all the aged samples. Although the negative charge tends to spread across the sample, more charge can be found in the region adjacent to the electrodes. The total amount of charge accumulated in the sample was calculated by integrating the modulus of the charge density across the sample thickness.

The relationship between the total amount of accumulated charge and the ageing time is illustrated in Fig. 3.

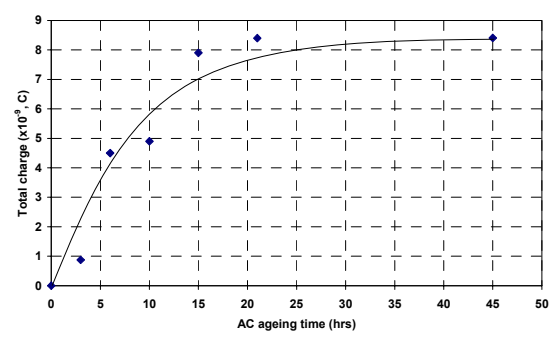

Fig. 3. Relationship between the total charge and ageing time at $50 \mathrm{kV} / \mathrm{mm}$.

It is evident that the amount of charge stored in the sample increases with the ageing time initially and then reaches a plateau after $20 \mathrm{hrs}$.

\section{Effect of Electrodes}

To verify the interface charge injection at ac stress, samples prepared with gold-coated electrodes (by cold sputtering) on both sides were also stressed for various periods of time and the space charge measurements are presented in Fig. 4. In comparison with the results shown in Fig. 2 where copper brass and semicon electrodes were used, it is noticed that the gold electrode has ability of blocking charge injection across the interface, resulting in very small space charge accumulation in the bulk of LDPE.

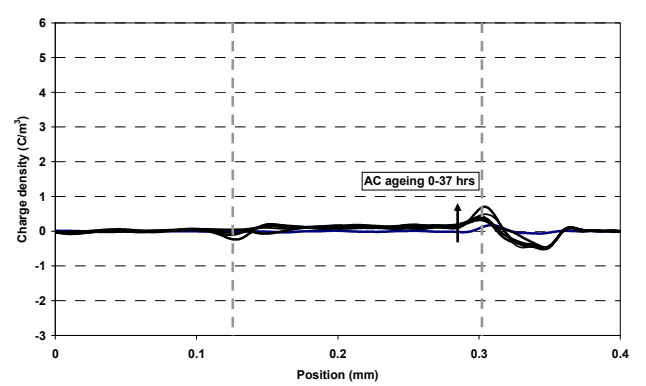

Fig. 4. Space charge accumulation in LDPE with gold electrodes

\section{Space Charge Characteristics under DC Voltage}

Apart from the observation of noticeable amount of space charge over ac ageing process, space charge dynamic characteristics under dc stress was also studied in the ac aged samples in order to explore the effect brought in by electrical ageing. In this test, space charge accumulation at de voltage of $10 \mathrm{kV}$ and its decay after the removal of applied voltage was compared with the fresh sample.

To summarise the results obtained under dc voltage the total charge accumulated in the samples is plotted as a function of stressing time for voltage on and decay time for voltage off respectively as shown in Figs. 5 and 6.

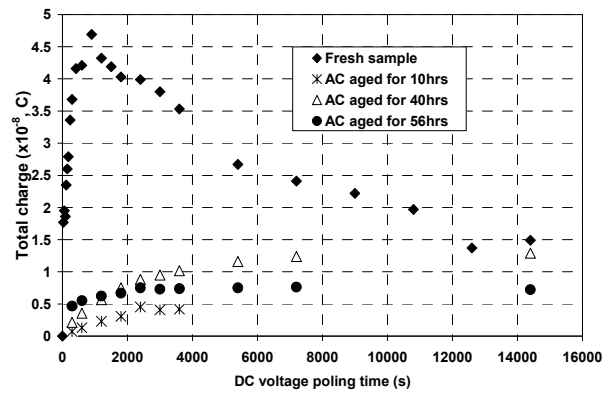

Fig. 5 Total amount of charge accumulation in fresh and aged samples with DC stress application

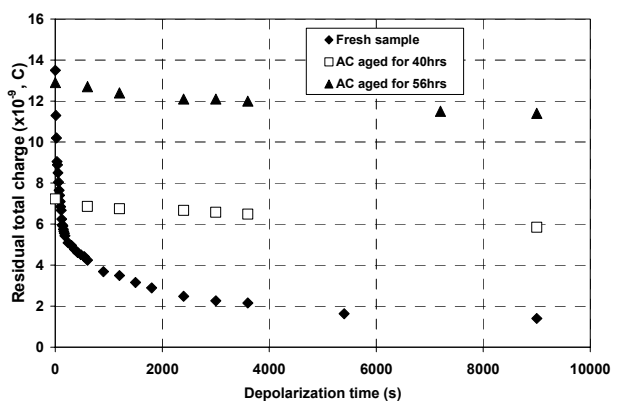

Fig. 6. Charge decay in fresh and aged samples

From Figs. 5 and 6, it is clear that the fresh sample shows a more active charge movement, both in charge accumulation and charge decay, in comparison with the aged samples. Space charge in the fresh sample reached its maximum within 15 minutes $(\sim 1000 \mathrm{~s})$ after the dc 
stressing voltage was applied and then gradually decreased in the magnitude until the arrival of the equilibrium due to the combination of packet charge in the material bulk. The space charge in the aged samples present a slow accumulating rate and reaches its steady status with the same magnitude as that in the fresh sample. Similar phenomenon is also observed in the unaged sample in the decay test, in which space charge accumulated during poling stage decreased quickly in the first 4 or 5 minutes and then showed a slow decay rate, whereas the aged samples, in contrast, presented a very stable charge stored previously in the decay test with the removal of the poling voltage. A typical example is illustrated in figure 7 . The results qualitatively agree with the research carried out by Mazzanti et. al $[14,15]$ in which they noticed that space charge decreased slower and slower with the ageing time increased in the sample of LDPE. This discovery may suggest that more deeper-traps have been created, resulting in considerable amount of space charge in ac ageing test. As a result of charge initiation at ac stress, the fresh and aged samples presented different space charge equilibrium distributions after dc poling.

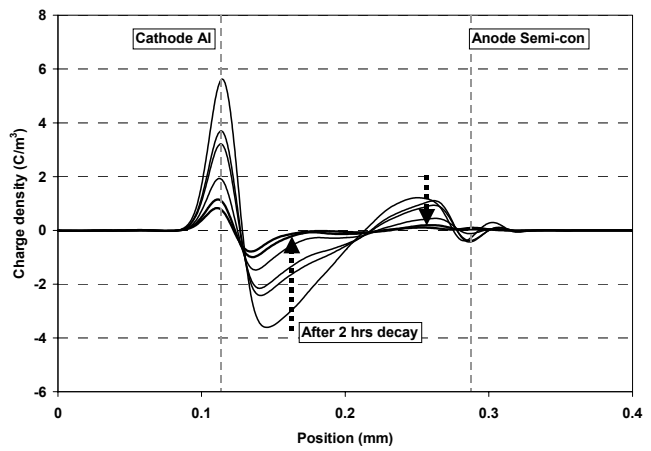

(a) Unaged sample

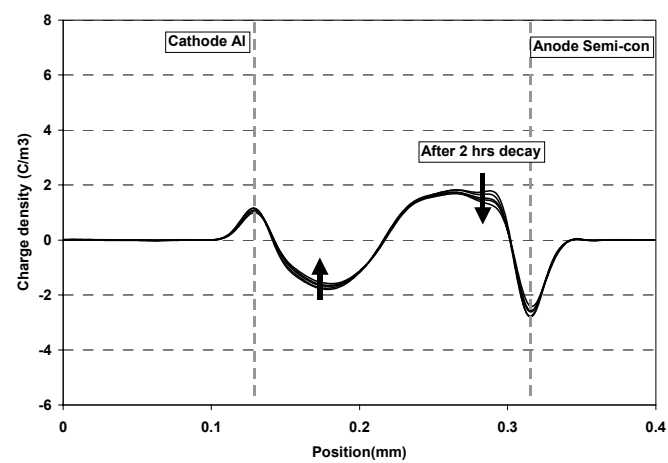

(b) Aged at ac field for 56 hours

Fig. 7. Space charge decay after DC poling

\section{Instrumental Analysis}

It is known that long-term ageing can cause chemical changes, which subsequently deteriorate electrical performance of the material. Fourier Transform Infrared (FTIR) and Raman spectra are believed very sensitive to the chemical degradation which may take place under the influence of electric stress. In the present study, theses techniques have been employed to monitor any chemical changes happened in the aged sample. Surprisingly, no significant change was observed from the results of FTIR and Raman spectra after the sample being aged 56 hours except a slightly rise in background of Raman spectrum of the aged sample. The rise is caused by fluorescence, which is due to partial degradation of the polymer [16].

\section{DISCUSSION}

Despite the increasing body of evidence of space charge presence under ac electric field in polymer insulation, the mechanism of charge generation and accumulation and its correlation with electrical ageing under ac stress is not yet clear. When the sample was stressed at $50 \mathrm{kV} / \mathrm{mm}$ under ac voltage the total charge within the bulk of the sample showed a clear increase with stressing time and then saturated after 20 hours. The deviation from the critical frequency of $0.01 \mathrm{~Hz}$ mentioned in literature [10] for space charge accumulation under ac voltage suggests that electric field in this case plays a key role in charge generation. As additive-free LDPE was selected in the present study, the impurity effect therefore should not be a major player in the charge generation. Space charge accumulated in the sample can be directly attributed to the charge injection at the dielectric and electrode interface. This conclusion has also been proved by the absence of space charge when the sample was coated on both sides with gold electrodes which possess low charge injection ability [17] because of its high work function.

The existence of trap and distribution in trap depth in a polymeric dielectric are the two main factors affecting charge accumulation. From the results presented it is believed that some amount of traps with a deeper energy level may have been generated in LDPE over the ageing process. Otherwise, it might not be able to observe charge presence if the quantities of charge injected and extracted at interfaces are equal in two half cycles. In Fig. 5, it is noticed that un-aged and aged LDPE samples possess obviously different charge accumulating tendency. Except the charge residual after AC stressing, aged samples showed a much lower space charge accumulation speed in comparison with the fresh sample in which space charge initiated very quickly as DC voltage was applied and then declined gradually to its steady status with time elapsing. This phenomenon is in accordance with the packet charge observed in the bulk of un-aged sample during poling time. The meeting of two charge fronts results in the recombination of opposite sign of charges. Fig. 8 displays the accumulation of space charge adjacent to the electrodes initially and then moves towards to the centre of sample giving a flat distribution. This result may imply that shallower-trapped charges dominate in the fresh sample; as a result, higher charge mobility is expected in decay after removal of poling voltage, as shown in Fig. 7(a). 
Whilst the electrical ageing has produced more deepertraps in the bulk of the LDPE, consequently, a slow space charge accumulation and decay rates, and high final net charge residual as well have been observed in the aged samples, see Fig. 7(b).

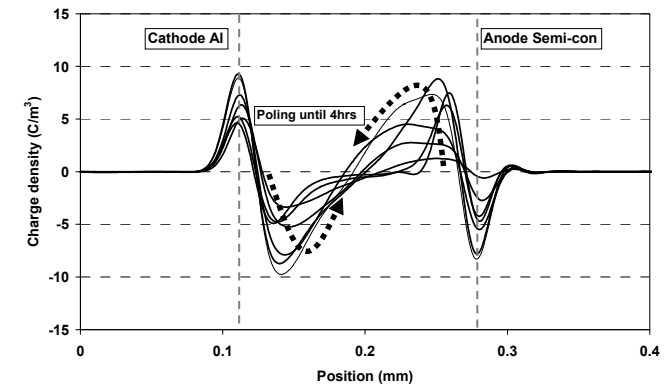

Fig. 8 Packet charge evolution in unaged sample at DC poling stress (with volts off)

\section{CONCLUSIONS}

This paper has presented experimental results of space charge accumulation under ac electric field and the effect of electrical ageing on charge behaviour when dc voltage is applied across LDPE samples.

After electrical ageing under ac stress of $50 \mathrm{kV} / \mathrm{mm}$, a considerable negative space charge is accumulated in the bulk of the material and the total amount of charge increases with the ageing time. Decay test indicates that the charges are captured in deep traps. This may suggest that some deep traps are generated over electrical ageing process. The comparison between samples with and without gold-coated electrode suggests that the charge generation under ac stress is mainly caused by the interface injection with the aid of considerably high electric stress.

The effect of electrical ageing on space charge activities is significant both in build-up (polarization) and decay (depolarization), which is believed to be controlled by trap density and depth. This type of alternation in trap characteristics cannot be distinguished by instrumental analysis, i.e. FTIR and Raman spectrum. This suggests that the space charge measurement can be developed as an effective technique to diagnose the early stage of polymer insulation ageing.

\section{REFERENCES}

[1] X. Wang, D. Tu, Y. Tanaka, T. Muronaka, T. Takada, C. Shinoda and T. Hashizumi, "Space charge in XLPE power cable under dc electrical stress and heat treatment”, IEEE Trans. Diel. Electr. Insul., Vol. 2, No. 3, pp. 467-474, 1995.

[2] B. Sanden, "Space charge accumulation in fresh and degassed XLPE insulation", 28th Diel. Soc. Meeting, pp225-233, Sept 1998.

[3] K. R. Bambery and R. J. Fleming, "The temperature dependence of space charge accumulation in cross- linked polyethylene", Journal of Thermal Analysis, Vol. 50, pp. 19-31, 1997.

[4] A. E. Davies and D. A. Hodgson, "Electrical ageing effects in polyethylene", $5^{\text {th }}$ Intel. Conf. DMMA, UK, IEE publication No. 289, pp. 254-257, 1988.

[5] L. A. Dissado, G. Mazzanti and G. C. Montanari, "The role of trapped space charges in the electrical aging of insulating materials", IEEE Trans. Diel. Electr. Insul., Vol. 4, No. 5, pp. 496-506, 1997.

[6] P. jr Notingher, A. Tourelle, J. Santana and M. Albertini, "Space charge in A.C.-poled XLPE for HV cables", Jicable 99, pp. 701-706, 1999.

[7] S. Agnel, A. Toureille, G. Platbrood and G. Geert, "Study of AC charging ability of XLPE insulating for power cable", Jicable 99, pp. 656-661, 1999.

[8] S. Hole, C. Alquié and J. Lewiner, "Space charge distribution determination under A.C. stress", C. R. Acad. Sci. Paris, t. 316, Série II, pp. 157-162, 1993.

[9] M. Mammeri, C. Laurent and J. Salon, "Influence of space charge build up on the transition to electrical treeing in PE under ac voltage", IEEE Trans. Diel. Electr. Insul., Vol. 2, No. 1, pp. 27-35, 1995.

[10] T. Takada, "Acoustic and optical methods for measuring electric charge distributions in dielectrics", IEEE Trans. Diel. Electr. Insul., Vol. 6, pp. 519-547, 1999.

[11] Y. F. F. Ho, G. Chen, A. E. Davies, S. G. Swingler, S. J. Sutton, R. N. Hampton, S. Hobdell, "Measurement of space charge in XLPE insulation under $50 \mathrm{~Hz}$ AC electric stresses using the LIPP method", IEEE Trans. Diel. \& Electr. Insul., Vol. 9 No 3, pp. 362-370, 2002.

[12] C. Bert, C. Heninion, J. Lewiner, C. Alquié, N. Hampton, J. Freestone and S. Verne, "Measurement of space charge distribution under $50 \mathrm{~Hz}$ A.C. stress", Jicable, pp. 195-199, 1995.

[13] G. Katsuta, A. Yoya, Y. Li, M. Okashita, F. Aida, Y. Ebinuma and Y. Ohki, "Experimental investigation on the cause of harmfulness of the blue water tree to XLPE cable insulation", IEEE Trans. on DEI., Vol. 6, pp. 887-891, 1999

[14] G. Mazzanti, G. C. Montanari and F. Palmieri, "Quantities extracted from space-charge measurement as Markers for Insulation Ageing”, IEEE Trans. DEL, Vol. 10, pp. 198-203, 2003

[15] G. Mazzanti, G. C. Montanari and J. M. Alison, "A Space-charge Based Method for Estimation of Apparent Mobility and Trap Depth as Markers for Insulation Degradation -Theoretical Basis and Experimental Validation", IEEE Trans. DEL, Vol. 10, pp. 187-197, 2003

[16] G. Chen, M. Fu and X.Z. Liu, "Influence of ac ageing on space charge dynamics in LDPE", $13^{\text {th }}$ ISH, Delft, Netherland, pp.365, 2003.

[17] G. Chen, T. Tay, A. E. Davies, Y. Tanaka and T. Takada, "Electrodes and Charge Injection in Lowdensity Polyethylene, Using the Pulsed Electroacoustic Technique", IEEE Trans. on DEI, Vol.8, 00. 867-873, 2001 